\title{
ANTIBIOTIC RESISTANCE PATTERN OF URINARY ISOLATES IN A TERTIARY CARE HOSPITAL IN BERHAMPORE, WEST BENGAL
}

\author{
Mohua Bose1, Ranjan Basu²
}

${ }^{1}$ Associate Professor, Department of Microbiology, Murshidabad Medical College, Berhampore, West Bengal, India.

${ }^{2}$ Assistant Professor, Department of Microbiology, Murshidabad Medical College, Berhampore, West Bengal, India.

\begin{abstract}
BACKGROUND
ABSTRACT

Urinary Tract Infection (UTI) is one of the commonest infections worldwide and a major public health problem. It may be caused by almost all known bacterial pathogens. A wide range of antibiotics are available for its treatment. In majority of the cases, antibiotic therapy is started empirically without waiting for the report of urine culture. Therefore, it becomes mandatory to determine the bacteriological profile of uropathogens in a particular region and their antimicrobial resistance pattern, so as to ensure the selection of an effective empirical treatment.
\end{abstract}

\section{MATERIALS AND METHODS}

This was a retrospective descriptive study. In this study of one-year duration, 3192 urine samples were processed. Age and gender were recorded from patients' data. Urine culture was performed by conventional methods and the isolates were identified by standard microbiological techniques and conventional biochemical methods. Antimicrobial susceptibility testing was done by Kirby-Bauer disc diffusion method.

\section{RESULTS}

703 samples yielded growth, of which $68.14 \%$ were from females and $31.86 \%$ were from males. The most commonly isolated pathogen was Escherichia coli (35.13\%) followed by Klebsiella spp. (27.88\%). Escherichia coli exhibited lowest resistance to Imipenem (1\%) followed by nitrofurantoin (19\%). Klebsiella spp. exhibited lowest resistance to Imipenem (1\%) followed by piperacillin/ tazobactam (27\%). The organisms were highly resistant to ticarcillin/ clavulanic acid (82\% - 85\%). Pseudomonas spp. showed highest resistance to ceftazidime (86.7\%). They were least resistant to piperacillin/ tazobactam (6.7\%) followed by Imipenem (13.3\%). Enterococcus spp. was least resistant to linezolid (2.2\%). They were highly resistant to Penicillin (92.8\%), aminoglycosides, macrolides, fluoroquinolones and tetracycline. $14.5 \%$ of isolates exhibited vancomycin resistance. Staphylococcus aureus isolates showed least resistance to Imipenem (2.6\%). 43.6\% of the isolates were vancomycin resistant.

\section{CONCLUSION}

As the distribution of urinary pathogens and their resistance pattern to antibiotics vary from one region to another, it is necessary to determine the most common uropathogens in a particular area and their resistance pattern so as to develop effective antibiotic policies and design a rational empirical therapy so that the best empirical therapy can be chosen, thus preventing the indiscriminate use of antibiotics and development of antimicrobial resistance.

\section{KEY WORDS}

UTI, Antimicrobial Susceptibility Testing, Antimicrobial Resistance Pattern, Uropathogens.

HOW TO CITE THIS ARTICLE: Bose M, Basu R. Antibiotic resistance pattern of urinary isolates in a tertiary care hospital in Berhampore, West Bengal. J. Evolution Med. Dent. Sci. 2018;7(31):3466-3471, DOI: 10.14260/jemds/2018/781

\section{BACKGROUND}

Urinary Tract Infection (UTI) is one of the most common causes of infection worldwide with an estimated 150 million cases per year and the second most common cause of visit to hospitals by patients.1,2 It is a major public health problem with a high morbidity rate and accounts for the highest healthcare cost among urological diseases. ${ }^{3}$ UTI includes a wide spectrum of diseases ranging from mild asymptomatic cystitis to pyelonephritis and septicaemia. ${ }^{4}$ The syndrome is commoner in females: approximately $50 \%$ of adult women experience at least one episode of UTI during their lifetime,

'Financial or Other Competing Interest': None.

Submission 19-06-2018, Peer Review 12-07-2018,

Acceptance 19-07-2018, Published 30-07-2018.

Corresponding Author:

Dr. Ranjan Basu,

Flat No. 3A, $3^{\text {rd }}$ Floor,

\#377 Kalikapur Road,

Kolkata-700078, West Bengal, India.

E-mail: microbiofaculty@gmail.com

DOI: $10.14260 /$ jemds $/ 2018 / 781$

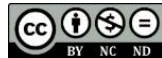

whereas men have a lifetime risk of only $13 \% .5,6,7$ This is probably because of the shorter length of the urethra in females, which facilitates entry of bacteria into the urinary tract easily. ${ }^{8}$ The incidence of uncomplicated UTI in healthy women is $50 / 1000 /$ year. ${ }^{9}$ UTI is most common in diabetic patients and patients with some structural and neurological abnormalities of the urinary tract, which interfere with the urine outflow. Instrumentation in the urinary tract like catheterisation and cystoscopy also predispose to UTI. UTI is also a leading cause of hospital acquired infection.

Almost all known bacterial pathogens have been implicated as possible causative agents of UTI.10,11 However, the most common isolate in uncomplicated UTI is Escherichia coli.5,6 Other organisms commonly implicated are Klebsiella spp., Proteus spp., Enterobacter spp., Pseudomonas spp., Staphylococcus aureus, Coagulase negative Staphylococcus and Enterococcus spp.

The introduction of antibiotic therapy has significantly contributed to the management of UTIs. Although, there is a large group of antimicrobial agents available for the treatment of UTIs, none of them can treat all UTIs. Moreover, 
in majority of the cases antibiotic therapy is started empirically without waiting for the report of urine culture from the Microbiology Laboratory. The distribution of urinary pathogens and their resistance pattern to antibiotics vary from one region to another.12,13 Therefore, it becomes mandatory to determine the bacteriological profile of uropathogens in a particular region and their antimicrobial susceptibility, so as to ensure the selection of an effective empirical treatment. ${ }^{14}$ Uropathogens have shown a change in pattern of susceptibility to antibiotics resulting in an increase in resistance to commonly used antibiotics. ${ }^{15}$ UTIs are usually treated with broad-spectrum antibiotics, although an antibiotic with a narrow spectrum of activity could have been appropriate due to concerns about infection with resistant organisms. The indiscriminate use of antibiotics has invariably led to development of resistance to many antimicrobial agents, which has become a major problem worldwide in the recent years. The aetiology of UTI and antibiotic resistance pattern of uropathogens have been changing over the past years. ${ }^{16}$ Therefore, a knowledge about the pathogens responsible for UTI and their resistance pattern may help clinicians to select the appropriate empirical treatment. ${ }^{17,14}$ This may also help to develop antibiotic policies in a health care facility.

We hereby undertook a study to determine the common urinary pathogens in our hospital and their antibiotic resistance pattern.

\section{Aims and Objectives}

The study was undertaken to determine the pathogens isolated from urine specimens and their resistance pattern to different antimicrobial agents.

\section{MATERIALS AND METHODS}

This is a retrospective descriptive study of one-year duration from May 2017 to April 2018 conducted in the Department of Microbiology in a tertiary care hospital in Berhampore in Murshidabad District of West Bengal. A total of 3192 urine samples received in the department during the study period from symptomatic cases were included in the study. The data concerning the age and gender were collected from patient's records.

Each sample was inoculated on blood agar and MacConkey media with a standard loop and was incubated at $37^{\circ} \mathrm{C}$ overnight. A growth of $\geq 10^{5}$ colony forming units $/ \mathrm{mL}$ was considered to be significant. The identification of the bacterial growth was done by Gram staining, standard microbiological techniques and conventional biochemical methods.

Antimicrobial susceptibility testing was done by KirbyBauer disc diffusion method on Mueller-Hinton agar and the interpretations were carried out as per Clinical and Laboratory Standards Institute (CLSI) guidelines.18,19 The control strains used were Escherichia coli ATCC 25922, Pseudomonas aeruginosa ATCC 27853 and Staphylococcus aureus 25922. The following antibiotics were tested for gram-negative bacilli: Imipenem (10 mcg), Meropenem (10 $\mathrm{mcg})$, piperacillin/ tazobactam $(100 / 10 \mathrm{mcg})$, ceftriaxone (30 mcg), cefotaxime (30 mcg), cefixime ( $5 \mathrm{mcg}$ ), cefepime (30 mcg), Cefoperazone (75 mcg), ceftazidime (30 mcg), gentamicin (10 mcg), amikacin (30 mcg), ciprofloxacin (5 mcg), ofloxacin (5 mcg), norfloxacin $(10 \mathrm{mcg})$, ticarcillin/clavulanic acid (75/10 mcg), nitrofurantoin (300 mcg), trimethoprim/sulfamethoxazole (co-trimoxazole) $(1.25 / 23.75 \mathrm{mcg})$, tetracycline $(30 \mathrm{mcg})$ and moxifloxacin (5 mcg). For gram-positive organisms the following antibiotics were tested: Imipenem (10 mcg), Linezolid (30 mcg), Vancomycin (30 mcg), nitrofurantoin (300 mcg), netilmicin (30 mcg), azithromycin (15 mcg), erythromycin (15 mcg), gentamicin (10 mcg), amikacin (30 mcg), cefotaxime $(30 \mathrm{mcg})$, trimethoprim/sulfamethoxazole (co-trimoxazole) $(1.25 / 23.75 \mathrm{mcg})$, ciprofloxacin $(5 \mathrm{mcg})$, levofloxacin (5 mcg), penicillin (10U), tetracycline $(30 \mathrm{mcg})$ and cloxacillin (10 $\mathrm{mcg}$ ) (for S. aureus). Dehydrated media were procured from Hi-Media and antibiotic discs from Microexpress. All the analyses were performed using simple counts and percentage method.

\section{RESULTS}

Of the total 3192 urine samples cultured, 2101 (65.82\%) were from females and 1091 (34.18\%) were from males. Out of the total 3192 samples, 703 (22.02\%) samples yielded growth [Figure 1].

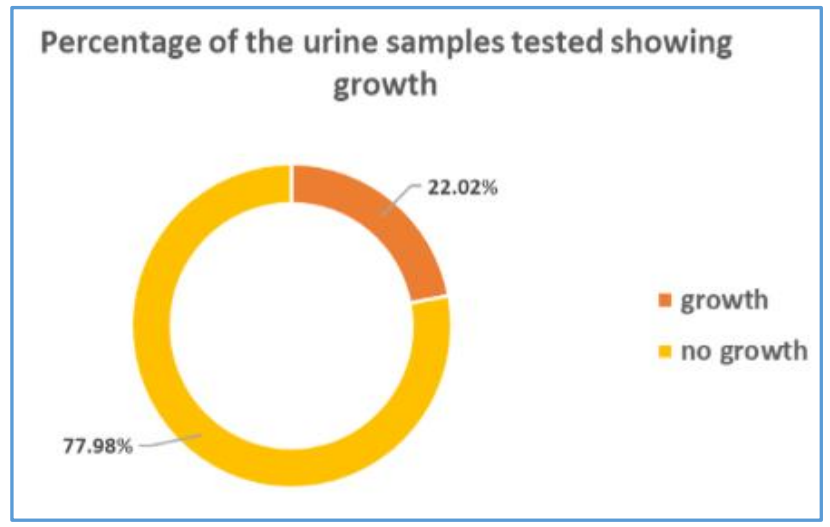

Figure 1. Growth from Urine Samples Tested

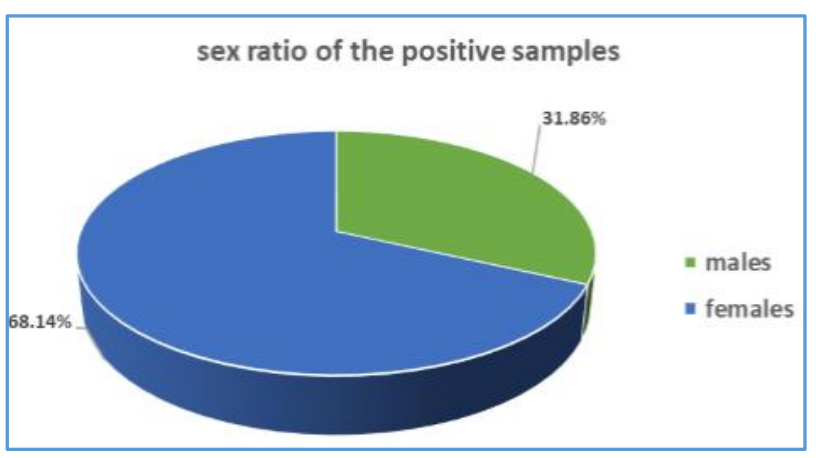

Figure 2. Sex Ratio of the Positive Samples

Among the positive cultures, 479 (68.14\%) were from females and 224 (31.86\%) were from males [Figure 2].

It was observed that highest number of subjects with positive culture was below 10 years age and the least number were those above 70 years [Table 1]. 


\begin{tabular}{|c|c|c|}
\hline Age in Years & No. & Percentage \\
\hline$<10$ & 213 & 30.30 \\
\hline $10-25$ & 170 & 24.18 \\
\hline $26-40$ & 156 & 22.19 \\
\hline $41-55$ & 89 & 12.66 \\
\hline $56-70$ & 58 & 8.25 \\
\hline$>70$ & 17 & 2.42 \\
\hline \multicolumn{2}{|c|}{ Table 1. Age distribution of Positive Samples } \\
\hline
\end{tabular}

\begin{tabular}{|c|c|c|}
\hline Organism Isolated & No. & Percentage \\
\hline Escherichia coli & 247 & 35.13 \\
\hline Klebsiella spp. & 196 & 27.88 \\
\hline Citrobacter spp. & 6 & 0.85 \\
\hline Proteus spp. & 14 & 1.99 \\
\hline Pseudomonas spp. & 15 & 2.13 \\
\hline Acinetobacter spp. & 3 & 0.43 \\
\hline Enterococcus spp. & 138 & 19.63 \\
\hline $\begin{array}{l}\text { Staphylococcus } \\
\text { aureus }\end{array}$ & 78 & 11.10 \\
\hline CONS & 1 & 0.14 \\
\hline Candida spp. & 5 & 0.71 \\
\hline Total & 703 & 100 \\
\hline \multicolumn{3}{|c|}{$\begin{array}{c}\text { Table 2. The Organisms Isolated from the Positives } \\
\text { Cultures }\end{array}$} \\
\hline
\end{tabular}

It was observed that the highest number of positive cultures yielded Escherichia coli (35.13\%) followed by Klebsiella spp. (27.88 \%). Gram-negative organisms accounted for 481 cases (68.42\%), of which 463 (96.26\%) were from the Enterobacteriaceae family. Gram-positive organisms accounted for 222 cases (31.58\%) [Figure 3].

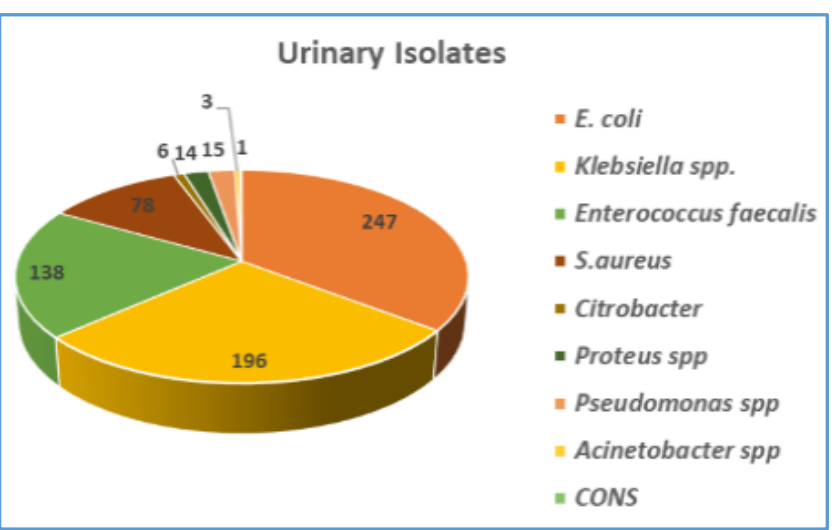

Figure 3. Urinary Isolates from Positive Cultures

\begin{tabular}{|c|c|c|c|c|c|c|c|c|c|c|c|c|c|c|c|c|c|c|c|}
\hline Organism & $\sum$ & 帘 & $\stackrel{N}{2}$ & 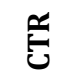 & 닌 & $\sum_{\text {넌 }}$ & $\sum_{U}^{i}$ & ڤે & ¿্ & 录 & 光 & 응 & 능 & ${ }_{z}^{x}$ & بِ & 它 & రั & 뇝 & $\sum^{\frac{1}{x}}$ \\
\hline E. coli & 1 & 20 & 30 & 69.6 & 62.8 & 73.7 & 71.7 & 83.4 & 80.6 & 74 & 49.4 & 70.9 & 57.9 & 60 & 82.2 & 19 & 72 & 54.3 & 85 \\
\hline $\begin{array}{l}\text { Klebsiella } \\
\text { spp. }\end{array}$ & 1 & 68.8 & 27 & 50.5 & 56.1 & 68.8 & 53.6 & 36.2 & 78.6 & 79.6 & 45.9 & 43.4 & 37.2 & 45.9 & 85.2 & 51.5 & 54.6 & 32.1 & 66.8 \\
\hline $\begin{array}{l}\text { Proteus } \\
\text { spp. }\end{array}$ & 0 & 0 & 21.4 & 35.7 & 50 & 57.1 & 69.4 & 45 & 79.3 & 64.2 & 64.2 & 42.9 & 32.2 & 78.6 & 78.6 & 42.9 & 64.2 & 57.1 & 57.1 \\
\hline $\begin{array}{c}\text { Citrobacter } \\
\text { spp. }\end{array}$ & 0 & 29.6 & 20 & 37.2 & 50 & 59.6 & 62.8 & 42.9 & 78.6 & 66.7 & 50 & 66.7 & 44.6 & 72.7 & 74.9 & 20 & 50 & 50 & 64.3 \\
\hline $\begin{array}{c}\text { Pseudomonas } \\
\text { spp. }\end{array}$ & 13.3 & 33.3 & 6.7 & 45.7 & 53.3 & 54.6 & 60 & 45.9 & 86.7 & 33.3 & 20 & 33.3 & 50 & 69.4 & 73.3 & 28.4 & 74.9 & 52.6 & 69.7 \\
\hline $\begin{array}{c}\text { Acinetobacter } \\
\text { spp. }\end{array}$ & 0 & 33.3 & 33.3 & 33.3 & 33.3 & 33.3 & 33.3 & 33.3 & 66.7 & 33.3 & 66.7 & 33.3 & 66.7 & 66.7 & 100 & 66.7 & 100 & 66.7 & 66.7 \\
\hline
\end{tabular}

Table 3. Antibiotic Resistance Pattern (\%) of Gram-Negative Bacilli. Here, IMP: imipenem, MRP: meropenem, PTZ: piperacillin/tazobactam, CTR: ceftriaxone, CTX: cefotaxime, CFM: cefixime, CPM: cefepime, CPZ: Cefoperazone, CAZ: ceftazidime, GEN: gentamicin, AK: amikacin, CIP: ciprofloxacin, OF: ofloxacin, NX: norfloxacin, TCC: ticarcillin/clavulanic acid, NIT: nitrofurantoin, COT: co-trimoxazole, TE: tetracycline, MXF: moxifloxacin.

It was observed that Escherichia coli isolates exhibited lowest resistance to Imipenem (1\%) followed by nitrofurantoin, meropenem and piperacillin/tazobactam. Klebsiella spp. isolates exhibited lowest resistance to Imipenem (1\%) followed by piperacillin/tazobactam. The organisms were highly resistant to ticarcillin/clavulanic acid. Both Escherichia coli and Klebsiella spp. were moderately resistant to the commonly used cephalosporins, fluoroquinolones and gentamycin. Pseudomonas spp. showed highest resistance to ceftazidime $(86.7 \%)$. They were most sensitive to piperacillin/tazobactam followed by Imipenem and Amikacin [Figures 4, 5 and 6].

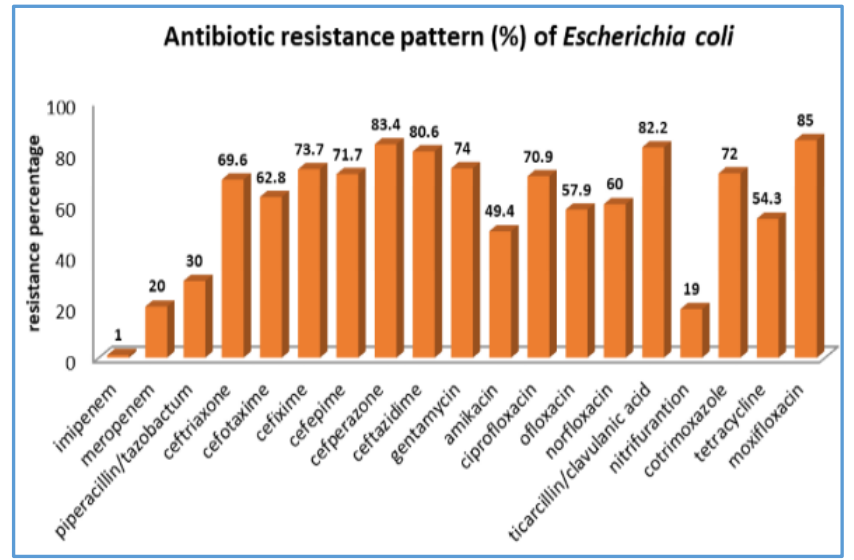

Figure 4. Antibiotic Resistance Pattern (\%) of Escherichia coli 


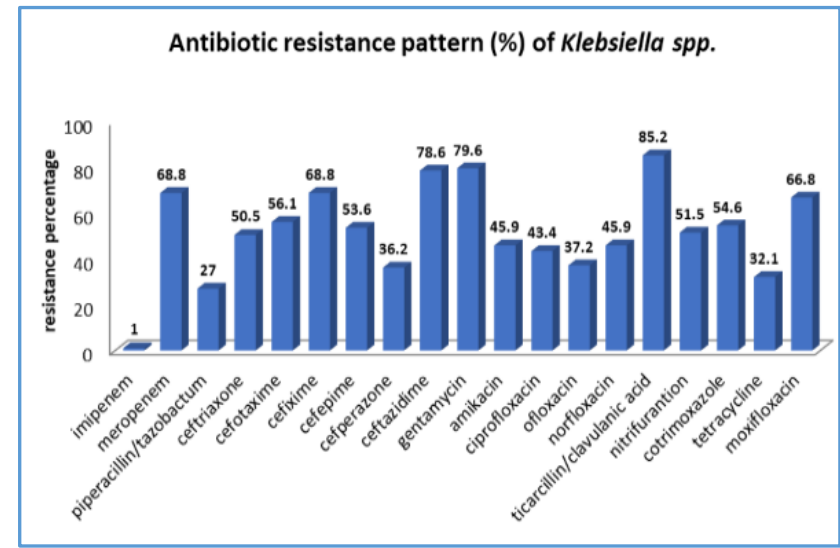

Figure 5. Antibiotic Resistance Pattern (\%) of Klebsiella spp.

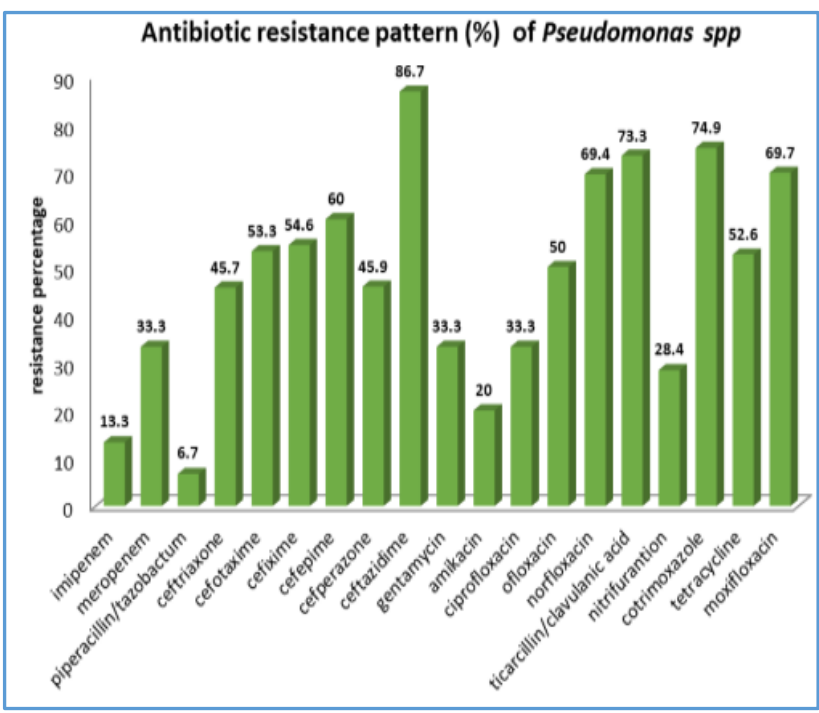

Figure 6. Antibiotic Resistance Pattern (\%) of Pseudomonas spp.

\begin{tabular}{|c|c|c|c|c|c|c|c|c|c|c|c|c|c|c|c|c|}
\hline Organism & IPM & LZ & VA & NIT & NET & AZM & $\mathbf{E}$ & AK & GEN & CTX & COT & CIP & LE & $\mathbf{P}$ & TE & COX \\
\hline $\begin{array}{c}\text { Enterococcus } \\
\text { spp. }\end{array}$ & 28.2 & 2.2 & 14.5 & 7.2 & 48.6 & 88.4 & 89.9 & 86.2 & 86.9 & 70.3 & 64.5 & 84.8 & 79.7 & 92.8 & 83.3 & - \\
\hline S. aureus & 2.6 & 5.1 & 43.6 & 11.5 & 7.7 & 73 & 93.6 & 19.2 & 59 & 55.1 & 76.9 & 70.5 & 55.1 & 86.4 & 47.4 & 70.5 \\
\hline CONS & 0 & 100 & 100 & 0 & 100 & 100 & 100 & 0 & 100 & 0 & 0 & 100 & 100 & 100 & 100 & 100 \\
\hline
\end{tabular}

Table 4. Antibiotic Resistance Pattern (\%) of Gram-positive cocci, Here, IMP: imipenem, LZ: Linezolid, VA: Vancomycin, NIT: nitrofurantoin, NET: netilmicin, AZM: azithromycin, E: erythromycin, GEN: gentamicin, AK: amikacin, CTX: cefotaxime, COT: co-trimoxazole, CIP: ciprofloxacin, LE: levofloxacin, P: penicillin, TE: tetracycline, COX: cloxacillin.

Among the Gram-positive organisms Enterococcus spp. was least resistant to linezolid $(2.2 \%)$ followed by nitrofurantoin. They were highly resistant to Penicillin (92.8\%), aminoglycosides, macrolides, fluoroquinolones and tetracycline. $14.5 \%$ of isolates were resistant to vancomycin. Staphylococcus aureus isolates showed least resistance to imipenem (2.6\%) followed by linezolid. $43.6 \%$ of the isolates exhibited resistance to vancomycin [Figures 7 and 8]. The only CONS isolate was sensitive only to Imipenem, nitrofurantoin, Amikacin, ceftriaxone and cotrimoxazole.

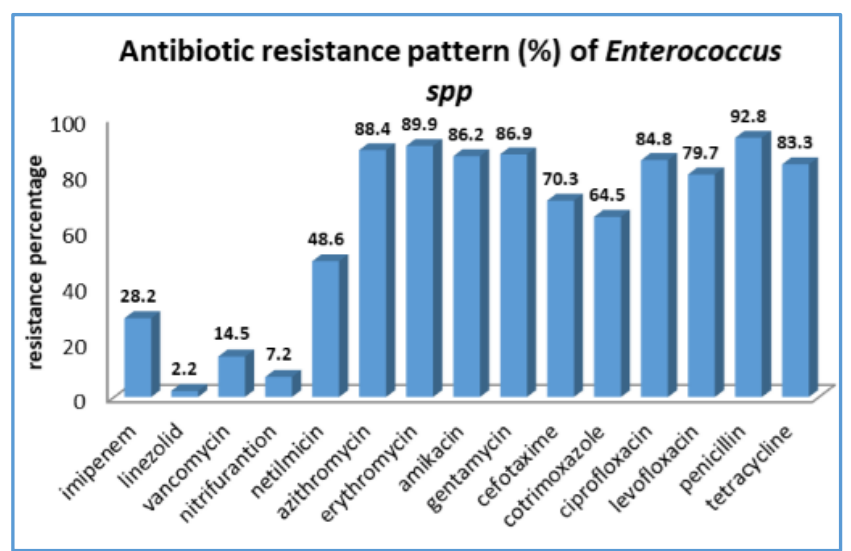

Figure 7. Antibiotic Resistance Pattern (\%) of Enterococcus spp.

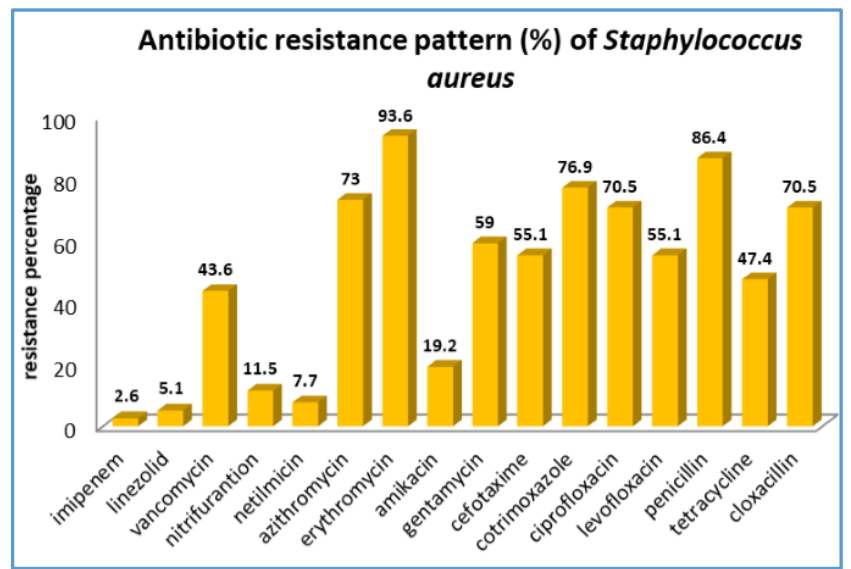

Figure 8. Antibiotic Resistance Pattern (\%) of Staphylococcus aureus

\section{DISCUSSION}

UTI is one of the commonest syndromes encountered by practitioners. This study was undertaken to determine the common uropathogens from cases of UTI and their antibiotic resistance pattern.

In our study, UTI was reported from $65.82 \%$ females and $34.18 \%$ males. This was similar to the findings by Sood S et al, who reported UTI from $37.67 \%$ males and $62.42 \%$ females. ${ }^{20}$ Female preponderance was reported from various studies: $57.74 \%$ by Kumar A et al, $70.5 \%$ by Hasan AS et al and 39.9\% females compared to $9.1 \%$ males by Semwal AC et al.21,22,23 
However, Patil $\mathrm{S}$ et al reported a slight male preponderance (57\%). ${ }^{24}$ The female preponderance is probably related to the short length of the female urethra and its proximity to the anal opening facilitating entry of bacteria into the urinary tract easily. 8

The most commonly isolated organism in our study was Escherichia coli (35.13\%) followed by Klebsiella spp. (27.88\%). This is in accordance with studies by Kumar A et al and Hasan AS et al, where Escherichia coli accounted for $37.41 \%$ and $50.7 \%$ of isolates followed by Klebsiella spp. accounting for $32.79 \%$ and $27.6 \%$ respectively. ${ }^{21,22}$ Similar results were also reported by Ranjbar $\mathrm{R}$ et al, Amin $\mathrm{M}$ et al, Tankhiwale SS et al and Somashekara SC et al.25-28 On the contrary, Chan RK et al found Klebsiella spp. (25\%) to be more prevalent than Escherichia coli (17.7\%). ${ }^{29}$

Among the Gram-positive organisms, the commonest isolate in our study was Enterococcus spp. (19.63\%) followed by Staphylococcus aureus (11.10\%), whereas Hasan AS et al and Semwal AC et al and reported more Staphylococcus aureus than Enterococcus spp. in their studies. ${ }^{22,23}$ In the present study, only one Coagulase-negative Staphylococcus (CONS), was isolated. The differences in the frequency of isolation of uropathogens in different studies from different geographic areas reflects the influence of various demographic factors.

In our study, the members of the Enterobacteriaceae family showed least resistance to Imipenem $(1 \%$ for both Escherichia coli and Klebsiella spp.). Imipenem was also reported as the least resistant antibiotic by Kumar A et al ( 0 $1.76 \%)$, Somashekara SC et al (14.7\%) and Manjunath GN et al $(7.5 \%) .{ }^{21,28,30}$ The organisms were highly sensitive to meropenem, piperacillin/tazobactam and nitrofurantoin except for the Klebsiella spp., which showed an unusually high resistance to meropenem (68.8\%) for unknown reasons. This is in concordance with studies by Kumar A et al and Semwal AC et al.21,23 Similar low resistance to nitrofurantoin was reported by Sood S et al and Patil S et al (15\%). ${ }^{20,24}$ This may be related to the low usage of Imipenem, meropenem and piperacillin/ tazobactam in the community. As for nitrofurantoin, this antibiotic has a narrow spectrum and hence limited indication and is highly concentrated in the urine, thereby retaining its activity in spite of many years of usage, especially against Escherichia coli. ${ }^{1}$ The organisms showed a moderate-to-high resistance to cephalosporins, aminoglycosides, fluoroquinolones, tetracycline, cotrimoxazole and ticarcillin/clavulanic acid. This may be due to the indiscriminate use of these antibiotics in the community for empirical treatment of UTI.

The Gram-positive organisms isolated in our study exhibited high resistance to penicillin (Enterococcus spp. 92.8\% and Staphylococcus aureus 86.4\%). Similar results were obtained by Hasan AS et al,(22) who reported $98.8 \%$ and $65.6 \%$ resistance to penicillin for Enterococcus spp. and Staphylococcus aureus respectively. Enterococcus spp. were least resistant to linezolid followed by nitrofurantoin. They were highly resistant to aminoglycosides, macrolides and fluoroquinolones as also reported by Hasan AS et al. ${ }^{22}$ Vancomycin resistance was reported in $14.5 \%$ of isolates as compared to $1.82 \%$ reported by Sood $S$ et al. ${ }^{20} 5.1 \%$ of Staphylococcus aureus exhibited resistance to linezolid compared to the $9.1 \%$ reported by Hasan AS et al.22 Vancomycin resistance was shown by $43.6 \%$ of
Staphylococcus aureus isolates similar to the study by Kumar A et al (43.87\%). ${ }^{21}$ In our study, imipenem resistance was reported in $28.2 \%$ of Enterococcus spp. and $2.6 \%$ of Staphylococcus aureus in contrast to $8.2 \%$ and $14 \%$ reported by Somashekara SC et al respectively. ${ }^{28}$ The rising resistance to vancomycin is a matter of great concern, as it is one of the few antibiotics available for treatment of resistant cases of Staphylococcus aureus and also Enterococcus spp.

\section{CONCLUSION}

Our study together with other studies from different geographic areas, highlight the importance of performing Antibiotic Susceptibility Test (AST) to determine the most effective antibiotics for the isolated uropathogens. Since the results of AST are not available to the practitioners immediately, clinicians have to depend on empirical choice of antibiotics. The distribution of urinary pathogens and their resistance pattern to antibiotics vary from one region to another. Therefore, it is necessary to determine the most common urinary isolates in a particular area and their local resistance pattern, so as to develop effective antibiotic policies and design a rational empirical therapy based on all the data of AST report, which would be somewhat customised to each geographic area. This will be helpful in choosing the best antibiotics for empirical therapy and avoiding the indiscriminate use of antimicrobial agents, which leads to selection of resistant mutants and emergence of drug resistance. Antibiotic resistance may be prevented by rotation of antibiotics, which is guided by a good hospital antibiotic policy. Imipenem still remains one of the most effective antibiotics for the treatment of infection caused by both Gram-positive and Gram-negative organisms.

\section{ACKNOWLEDGEMENT}

We extend our sincere thanks to all faculty members, technical and non-technical staffs of the Department of Microbiology of Murshidabad Medical College and Hospital for all the support given by them during the work period. We are thankful to Professor Manash Sarkar, Professor and Head of the Department, Microbiology for his continuous support extended to us during the study.

\section{REFERENCES}

[1] Karlowsky JA, Kelly LJ, Thornsberry C, et al. Trends in antimicrobial resistance among urinary tract infection isolates of Escherichia coli from female outpatients in the United States. Antimicrob Agents Chemother 2002;46(8):2540-5.

[2] Ronald AR, Pattullo AL. The natural history of urinary infection in adults. Med Clin North Am 1991;75(2):299-312.

[3] Gales AC, Sader HS, Jones RN, et al. Urinary tract infection trends in Latin American hospitals: Report from the SENTRY antimicrobial surveillance program (1997-2000). Diagn Microbiol Infect Dis 2002;44(3):289-99.

[4] Naveen R, Mathai E. Some virulence characteristics of uropathogenic Escherichia coli in different patient groups. Indian J Med Res 2005;122(2):143-7.

[5] Foxman B, Barlow R, D'Arcy H, et al. Urinary tract infection: self-reported incidence and associated costs. 
Ann Epidemiol 2000;10(8):509-15.

[6] Foxman B. Epidemiology of urinary tract infections: incidence, morbidity and economic costs. Am J Med 2002;113 Suppl 1A:5S-13S.

[7] De Backer D, Christiaens T, Heytens S, et al. Evolution of bacterial susceptibility pattern of Escherichia coli in uncomplicated urinary tract infections in a country with high antibiotic consumption: a comparison of two surveys with a 10 year interval. J Antimicrob Chemother 2008;62(2):364-8.

[8] Das RN, Chandrashekhar TS, Joshi HS, et al. Frequency and susceptibility profile of pathogens causing urinary tract infections at a tertiary care hospital in western Nepal. Singapore Med Journal 2006;47(4):281-5.

[9] Nicole W, Jon DM. Deciphering dysuria. Emerg Med 2008;40(9):29.

[10] Wilkie ME, Almond MK, Marsh FP. Diagnosis and management of urinary tract infection in adults. BMJ 1992;305(6862):1137-41.

[11] Bajaj JK, Karyakarte RP, Kulkarni JD, et al. Changing aetiology of urinary tract infections and emergence of drug resistance as a major problem. J Commun Dis 1999;31(3):181-4.

[12] Farrell DJ, Morrissey I, De Rubeis D, et al. A UK multicentre study of the antimicrobial susceptibility of bacterial pathogens causing urinary tract infection. J Infect 2003;46(2):94-100.

[13] Mathai D, Jones RN, Pfaller MA, et al. Epidemiology and frequency of resistance among pathogens causing urinary tract infections in 1,510 hospitalized patients: a report from the SENTRY Antimicrobial Surveillance Program (North America). Diagn Microbiol Infect Dis 2001;40(3):129-36.

[14] Smith RD, Coast J. Antimicrobial resistance: a global response. Bull World Health Organ 2002;80(2):12633.

[15] Magalit SL, Gler MT, Tupasi TE. Increasing antimicrobial resistance patterns of community and nosocomial uropathogens in Makati Medical Center. Philipp J Microbiol Assoc 2001;51:94-100.

[16] Kahan NR, Chinitz DP, Waitman DA, et al. Empiric treatment of uncomplicated urinary tract infection with fluoroquinolones in older women in Israel: another lost treatment option? Ann Pharmacother 2006;40(12):2223-7.

[17] Al-Jebouri MM, Mdish SA. Antibiotic resistance pattern of bacteria isolated from patients of urinary tract infections in Iraq. Open Journal of Urology 2013;3:124-31.
[18] Bauer AW, Kirby WM, Sherris JC, et al. Antibiotic susceptibility testing by a standardized single disk method. Am J Clin Pathol 1966;45(4):493-6.

[19] Clinical and Laboratory Standards Institute, Performance Standards for Antimicrobial Susceptibility Testing, $16^{\text {th }}$ Informational Supplement. M100-S16. Clinical and Laboratory Standard Institute. Wayne, PA: 2006.

[20] Sood S, Gupta R. Antibiotic resistance pattern of community acquired uropathogens at a tertiary care hospital in Jaipur, Rajasthan. Indian J Community Med 2012;37:39-44

[21] Kumar A, Kumar R, Gari M, et al. Antimicrobial susceptibility pattern of urine culture isolates in a tertiary care hospital of Jharkhand, India. Int J Basic Clin Pharmacol 2017;6(7):1733-9.

[22] Hasan AS, Nair D, Kaur J, et al. Resistance patterns of urinary isolates in a tertiary Indian hospital. J Ayub Med Coll Abbottabad 2007;19(1):39-41.

[23] Semwal AC, Mathuria YP, Saklani P. Study of antibiotic resistance pattern in uropathogens at a tertiary care hospital. Ann Int Med Den Res 2017;3(5):MB01-MB06.

[24] Patil S, Mahale K, Shetty P, et al. Antibiotic susceptibility pattern of urinary isolates from a tertiary care hospital with special reference to Gram negative bacteria. IOSR Journal of Dental and Medical Sciences 2013;12(1):49-51.

[25] Ranjbar R, Haghi-Ashtaini MT, Jafari NJ, et al. The prevalence and antimicrobial susceptibility of bacterial uropathogens isolated from paediatric patients. Iranian J Pub Health 2009;38(2):134-8.

[26] Amin M, Mehdinejad M, Pourdangchi Z. Study of bacteria isolated from urinary tract Infections and determination of their susceptibility to antibiotics. Jundishapur J Microbiol 2009;2(3):118-23.

[27] Tankhiwale SS, Jalgaonkar SV, Ahamad S, et al. Evaluation of extended spectrum beta lactamase in urinary isolates. Indian J Med Res 2004;120(6):553-6.

[28] Somashekara SC, Deepalaxmi S, Jagannath N, et al. Retrospective analysis of antibiotic resistance pattern to urinary pathogens in a tertiary care hospital in south India. J Basic Clin Pharm 2014;5(4):105-8.

[29] Chan RK, Lye WC, Lee EJ, et al. Nosocomial urinary tract infection: a microbiological study. Ann Acad Med Singapore 1993;22(6):873-7.

[30] Manjunath GN, Prakash R, Annam V, et al. Changing trends in the spectrum of antimicrobial drug resistance pattern of uropathogens isolated from hospitals and community patients with urinary tract infections in Tumkur and Bangalore. Int J Biol Med Res 2011;2(2):504-7. 